\title{
REVIEWS
}

\section{Corollary discharge across the animal kingdom}

\section{Trinity B. Crapse and Marc A. Sommer}

Abstract | Our movements can hinder our ability to sense the world. Movements can induce sensory input (for example, when you hit something) that is indistinguishable from the input that is caused by external agents (for example, when something hits you). It is critical for nervous systems to be able to differentiate between these two scenarios. A ubiquitous strategy is to route copies of movement commands to sensory structures. These signals, which are referred to as corollary discharge (CD), influence sensory processing in myriad ways. Here we review the $C D$ circuits that have been uncovered by neurophysiological studies and suggest a functional taxonomic classification of $C D$ across the animal kingdom. This broad understanding of CD circuits lays the groundwork for more challenging studies that combine neurophysiology and psychophysics to probe the role of CD in perception.
Receptor (or sensor) A sensory end organ that detects changes in the external world or the internal viscera.

\section{Effector}

An organ that becomes active in response to a nerve signal.

\section{Afferent}

A neuronal projection that conveys information to a structure. The term is often used in reference to sensory channels.
Department of Neuroscience, Center for the Neural Basis of Cognition, and Center for Neuroscience at the University of Pittsburgh, University of Pittsburgh, Pittsburgh, Pennsy/vania 15260, USA.

Correspondence to T.B.C. e-mail: tbc6@pitt.edu doi:10.1038/nrn2457
An animal that never moved could possess a relatively simple sensory system. It would need receptors to detect environmental changes, and a nervous system capable of interpreting the information. The animal would be limited by two main factors: the range of energy changes it could detect and the sophistication of the subsequent analyses it could perform. Within these limits, its sensory receptors should reliably detect near (for example, involving physical contact) and distant (for example, involving light) environmental occurrences.

Once an animal moves, however, the situation changes drastically. Movements contribute to forming a sense of space, as discussed by Poincaré ${ }^{1}$, and to improved, discriminative sensory perception. But movements have a potential downside as well: they introduce ambiguity about the source of sensory input. A newly mobile animal would be unable to determine whether a disturbance registered by its sensory receptors was the result of a change in the environment or simply a consequence of its own movement. As it walked, it might not know whether sudden activation of its skin receptors was due to a predator's paw or to an inanimate obstacle in its path, or whether detected changes in light and shade were caused by movements of external objects or by movements of its own photosensory organs.

\section{The cause of sensory input: self or other?}

The fundamental distinction concerning the origin of sensory input was discussed by von Holtz and Mittelstaedt ${ }^{2}$. They termed input that results from occurrences in the environment as "exafference"; this is the only kind of input that a stationary animal would experience. Exafference contrasts with "reafference", which refers to those inputs that inevitably result from an animal's own movements. Reafference is action-dependent and thus would be absent in immobile animals.

Sensory receptors are indifferent to the cause of their activation, so in principle they would convey both exafference and reafference equally, and downstream processing would proceed identically for both. However, von Holst and Mittelstaedt ${ }^{2}$ pointed out that this would lead to a manifold sensory and interpretative problem: reafference would be confused for exafference. This potential confusion would be particularly significant for the special abilities of some animals. For example, bats emit sounds that reflect back to them, but the reafferent echoes are mingled with exafferent noise from other sources (including nearly identical biosonar probes from other bats). Furthermore, informational ambiguity is only one facet of the underlying problem. Motor action can be quite violent to receptors, many of which are located on or near effectors (for example, muscles), and so movements could desensitize the primary afferents of a particular sensory channel. The animal would thus be unresponsive to independently occurring inputs that followed jarring motor acts.

Because the sensory problems that are introduced by movements are potentially devastating, any ancestral species that failed to solve them would have faced an evolutionary disadvantage. Those species that survived seem to have overcome the problem with a remarkably uniform mechanism: the animals' nervous systems keep 
Sensory processing stream The series of neuronal areas that are involved in analysing the information acquired by sense organs.

\section{Efferent}

A neuronal projection that conveys information away from a structure. The term is often used when referring to motor commands.

Decussation

The point where an axon or a pathway crosses another.

Phylogeny

The evolutionary development or history of a group of organisms, often depicted in family trees. track of their movement commands and inform the sensory processing stream about movements that are imminent. In the terminology of von Holtz and Mittelstaedt, the animal sends an "efference copy signal" - that is, a copy of the efferent motor command issued to an effector - to the sensory pathway. Thus, the combination of the input received from the sensory receptors and the motor copy that indicates how a movement might influence the input signal allows a moving animal to resolve the confusion in sensory input.

FIGURE 1a shows the basic circuit in which a sensor and a series of steps along a sensory pathway convey the exafferent and reafferent sensory signals to a motor pathway that impinges on the muscles to produce the appropriate movement. A branch from the motor pathway to the sensory pathway provides the efference copy. The general strategy involves the coincident production of a motor command destined for an effector and a motorcommand copy destined for a sensory structure. On receipt of the copy signal, the sensory structure adjusts appropriately to minimize, eliminate or compensate for the sensory consequences of the movement.

The term 'efference copy' implies an actual copy of the motor command (the efference) that targets the muscles; this term seemed appropriate for the questions that von

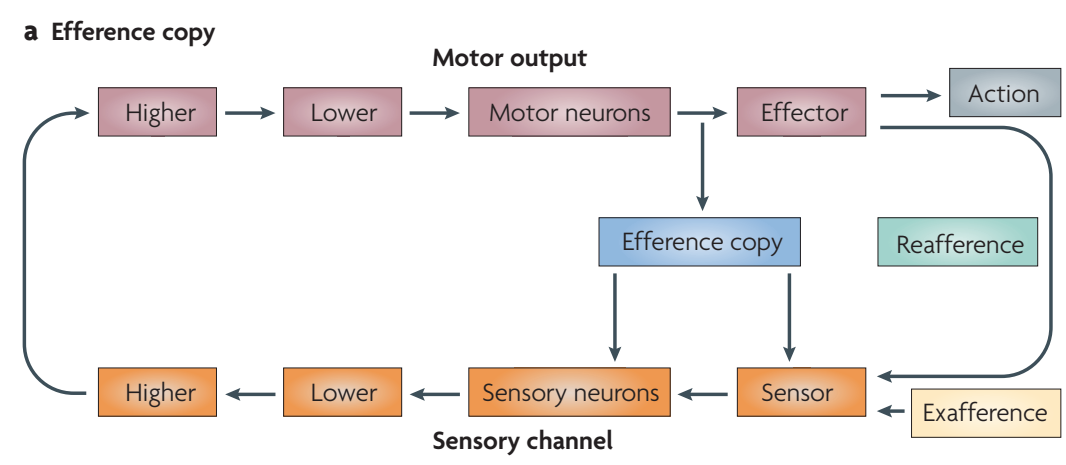

b Corollary discharge

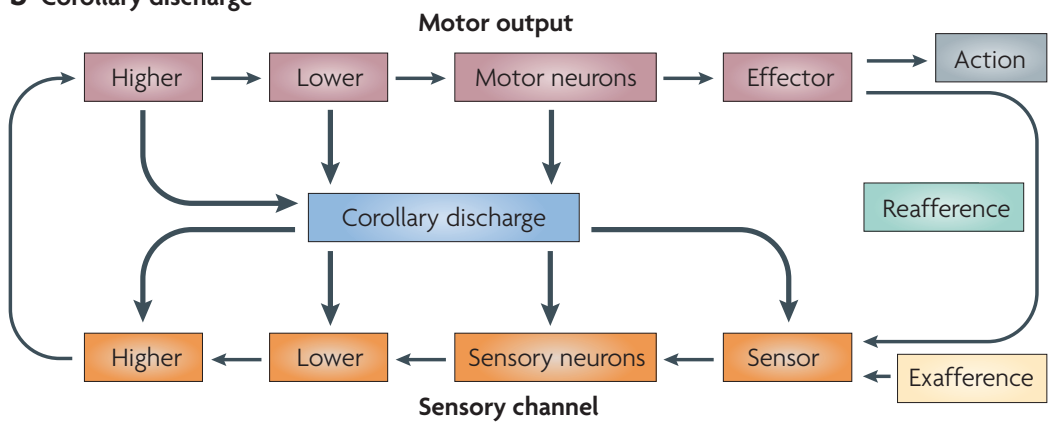

Figure 1 | Efference copy versus corollary discharge. a |A schematic of a sensorimotor circuit composed of a sensory pathway (shown in orange) and a motor pathway (shown in brown). Each pathway consists of a number of tiers that represent the complexity of the processing that has been performed and the distance from the periphery. A branch from the motor pathway to the sensory pathway (shown in blue) provides the efference copy. $\mathbf{b}$ |Corollary discharge. Motor-to-sensory signals are not confined to exact copies that target early tiers of the sensory channel. They can arise from almost all levels of the motor pathway and can target any tier of the sensory processing stream. These signals are known as corollary discharges (shown as thick arrows). Schematic inspired by von Holst and Mittelstaedt².
Holtz and Mittelstaedt were addressing in invertebrates and for the general analysis of sensory processing that takes place close to the motor output. However, it has become apparent that the decussation from motor to sensory areas might occur at any number of levels of motor control, some of which are remote from the final effector stage (FIG. 1 b). In studies on fish, Sperry ${ }^{3}$ coined the term "corollary discharge" (CD) to denote motor-related signals that influence sensory processing, but his conception was less specific as to where the branch from motor to sensory pathways should emerge. In this Review we compare motor-to-sensory circuits across different species and different levels of the nervous system, and so we use what seems to be the more general of the two terms: corollary discharge. At a mechanistic level CD can adopt one of multiple forms depending on how it is used: it can facilitate, inhibit or otherwise modulate its target.

Our main goal in this Review is to compare corollary systems across the animal kingdom. What is evident from experiments that have been performed over the past few decades is that multiple types of CD have evolved, and that each is particularly well suited to the problems faced by the species. Two recent reviews have summarized a few of them ${ }^{4,5}$. Here we provide a broader overview of the varieties of $\mathrm{CD}$ and place emphasis on two themes. First, we focus on critical functional differences, illustrated by the impact of $\mathrm{CD}$ on recipient sensory structures. Animals that occupy diverse niches show differences in their use of CD that, we propose, can be summarized with a functional taxonomy. Second, we consider the general similarities in CD circuits among animals. We find that CD circuits conform to a common neuronal plan, with only minor modifications in motor source and sensory termination. By classifying each circuit we attempt not only to summarize recent findings, but also to distill from them the core circuits for CD that they exemplify. To help illustrate our points, throughout this article we use FIG. $1 \mathrm{~b}$ and its colour-coded conventions as a template onto which each animal's particular pathway is represented. We close by discussing some implications of our current understanding of $\mathrm{CD}$ and considering several questions that remain unanswered.

\section{A taxonomy for CD}

As is made evident in this Review, CD lends itself to a taxonomic classification scheme (FIG. 2). Overall, CD can be dichotomized into lower- and higher-order functional (rather than phylogenetic) categories based on its 'operational impact' on the nervous system. The operational impact is the way in which the CD signal influences the recipient structure, with the aim of achieving sensorimotor harmony. The lower- and higher-order categories are subject to further subdivisions that represent the more specific functions of the signal.

Lower-order CD signalling is used for functions such as reflex inhibition and sensory filtration, both of which are examples of the control of sensation by the CNS. This type of CD serves a sentinel function by intervening at various points along a sensorimotor pathway to regulate the sensory information that enters the system (in the 


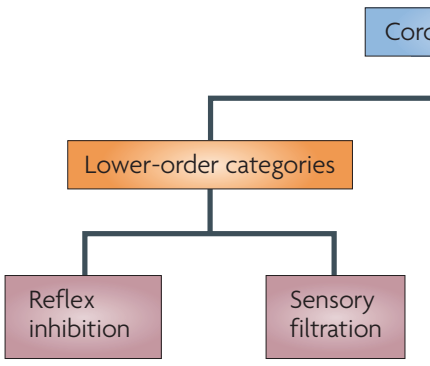

Central control of sensation orollary discharge

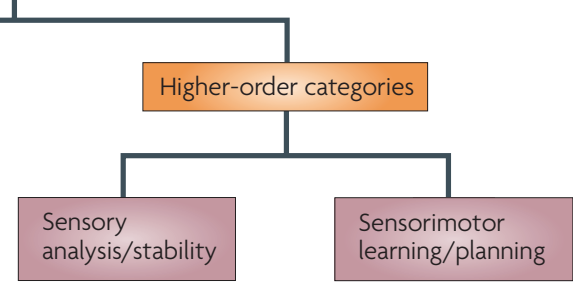

Central control of action and perception

Figure 2 | A taxonomic classification of corollary discharge. Corollary discharge can be classified globally into lower- and higher-order categories according to the function and operational impact of the signal. Lower-order functions include reflex inhibition and sensory filtration, which are examples of the control of sensation by the CNS. Higherorder functions include sensorimotor learning/planning and sensory analysis/stability, all of which are examples of the control of action and perception by the CNS.

Mechanoreceptor

A receptor that senses physical displacement.

Vestibular signal

A signal that conveys changes in head orientation, which are produced by head movements or changes in the position of the head with respect to gravity.

Proprioceptive signal A signal that conveys information about the position and movement of body parts.

Giant command neuron A motor neuron that is common in invertebrate species and that facilitates behaviours such as the rapidescape response. case of sensory filtration) and the motor responses that the information elicits (in the case of reflex inhibition). Such activity seems to be ubiquitous, as it is necessary for any animal that is equipped with sensory and motor systems. Most of the CD circuits that have been identified in invertebrates are of this variety.

Higher-order CD signalling participates in functions such as sensory analysis and stability, and sensorimotor planning and learning. On the perceptual side it facilitates the contextual interpretation of sensory information (analysis) and the construction and maintenance of an internal representation of this information (stability). On the sensorimotor side it facilitates novel motor-pattern acquisition (learning) and the execution of rapid movement sequences (planning). Examples of higher-order CD have been identified only in vertebrates thus far.

Keeping this taxonomic framework in mind, below we discuss the guises that lower- and higher-order CD signals assume in the nervous systems of various species. We first consider lower-order CD and investigate its role in functions related to the central control of sensation. Such functions are relatively peripheral operations that are carried out by almost every nervous system to control the initial inflow of sensory information.

\section{Lower-order CD}

Reflex inhibition. The nematode Caenorhabditis elegans (see FIG. 3a) has only 302 neurons $^{6}$ and a relatively simple behavioural repertoire, which nevertheless requires coordinative signalling by CD. Its avoidance response consists of two main reflexes: forward and backward progression ${ }^{7}$. These behaviours are antagonistic: when the nematode jolts forward owing to a tail stimulus, reafference from the head receptors would send it backwards (FIG. 3a). However, inhibitory neurons quickly silence the second reflex pathway and annul the effect of the reafference. Among sensory neurons, interneurons and motor neurons there are reciprocal patterns of connectivity that inhibit the inappropriate reflex whenever the opposite behaviour is activated ${ }^{8}$, a CD-like function. A comparable problem is faced by Xenopus laevis tadpoles during locomotion, and a CD regulatory circuitry remarkably similar to that of $C$. elegans modulates their avoidance reflexes ${ }^{9}$.

Gastropods must regulate their reflexes too, but in a different context from the above examples ${ }^{10}$ (FIG. 3b). Normally, gastropods such as Pleurobranchaea repel if contact is made with the tactile mechanoreceptors of its oral veil (its 'mouth'; FIG. 3b, schematic). However, this withdrawal response does not occur while the animal is feeding, despite there being comparable tactile stimulation. A CD of feeding commands, originating from a corollary discharge interneuron (CDI), was found to intervene during feeding. This effectively silences the appropriate withdrawal command neurons and inhibits maladaptive withdrawal from sustenance. For all the reflex-inhibition examples, these rudimentary $\mathrm{CD}$ circuits can be depicted in our organizing template as providing routes of contact between lower motor and lower sensory areas. These examples are thus instances of CD regulating sensation by reflex inhibition (FIG. 3c).

We have considered some specific cases of reflex coordination that involve the suppression, by $\mathrm{CD}$, of signals that would drive incompatible behaviours. Comparable circuits are present in more advanced organisms as well and have a prominent role in regulating the vestibular and proprioceptive signals that accompany voluntary movements (BOX 1).

Sensory filtration. Many organisms detect the presence of prey or the approach of predators by monitoring the energy that is captured by their hair-cell mechanoreceptors. These sensory systems detect mechanical perturbations that arise from events in the surrounding environment. One organism with a reliance on hair-cell sensors is the crayfish (see FIG. 4a). The crayfish responds to sudden and unpredicted events by tail-flipping to safety ${ }^{11}$. This escape response is triggered by information that enters the system through arrays of hair cells located on its tail and abdomen ${ }^{12-14}$. Signals related to water or air displacements are reported to a network of interneurons that synapse on to giant command neurons, which directly elicit the escape response. In order to escape only when it is most appropriate to do so, the crayfish must maintain afferent sensitivity for optimal threat detection while ignoring false escape cues arising from reafference. However, repeated reafference could result in habituation of the reflex, leaving the crayfish unresponsive to exafferent information.

CD mechanisms allow crayfish to move freely without inadvertently triggering or habituating their reflexive escape responses ${ }^{15}$. Activation of their hair cells normally causes a tail flip, but these same sensors are activated repeatedly during escape movements and thus must be regulated during escape. The circuitry contains CDIs that receive signals from the lateral giant command neurons, which initiate the escape response (FIG. 4a). The CDIs synapse on to interneurons (called primary afferent depolarizing interneurons) that presynaptically inhibit primary afferents from the tail hair cells. This arrangement prevents a dizzying feedback cycle of movement-escape that would otherwise ensue with each 
Teleception

Sensory reception that is specialized for the detection of distant external stimuli, such as light, sound and smell. movement. This example illustrates how CD signals that originate from a lower motor-control area target sensory neurons positioned at an early stage of the sensory processing stream (FIG. 4C). In terms of function, this circuit is almost identical to one that has been identified in the cockroach, another organism with a behaviourally important hair-cell/escape-response circuit ${ }^{16}$. In both of these animals, CD signals prevent reafferent saturation and inappropriate escape responses.

Fish face similar problems to the preceding invertebrate examples. The dogfish, for example, swims by generating rhythmic sinuous movements of its torso. These movements induce water turbulence that displaces the

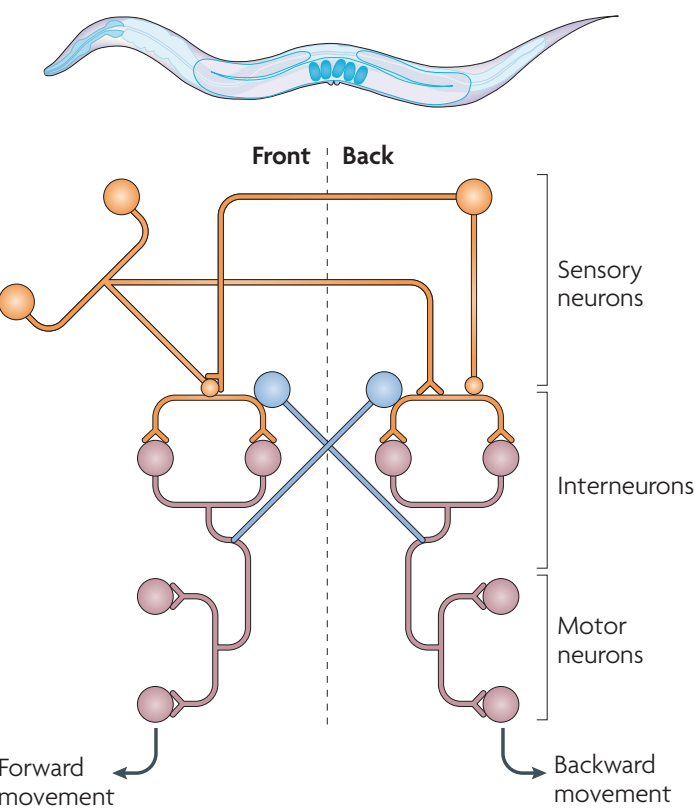

mechanosensitive hair cells of the lateral line system, a network of mechanoreceptors that line the cephalic and trunk regions ${ }^{17,18}$ and detect disturbances in the water column ${ }^{19}$. As with the cockroach and the crayfish, these hairs are stimulated by locomotion. To avoid reafferent saturation, CDs of swimming commands directly inhibit the lateral-line hair cells ${ }^{20}$. In this case the CD signals act directly on a sensor.

The preceding examples illustrate how CD of locomotion modulates pathways that are associated with contact and teleceptive mechanosensation. Next we assess the role of CD in regulating sensory pathways associated with two other teleceptive senses: audition and vision.

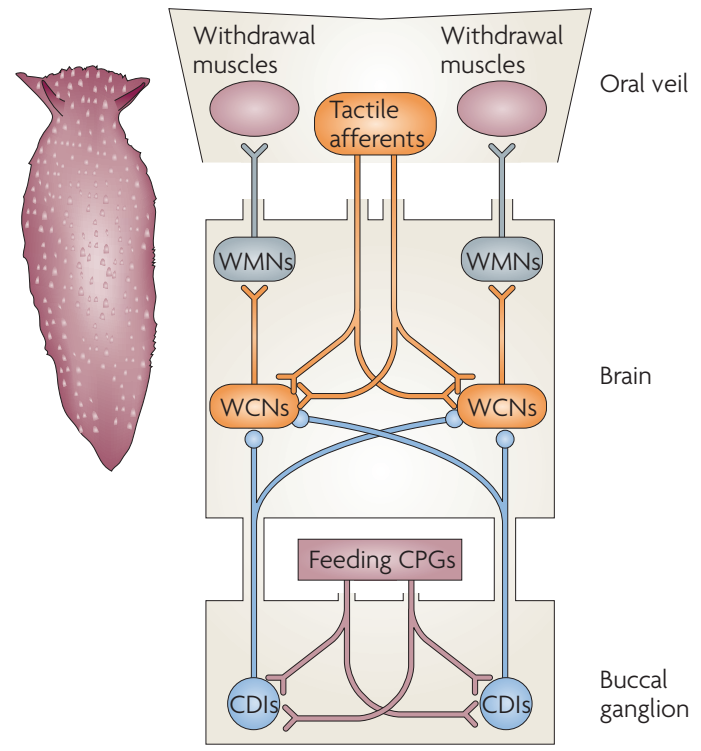

C

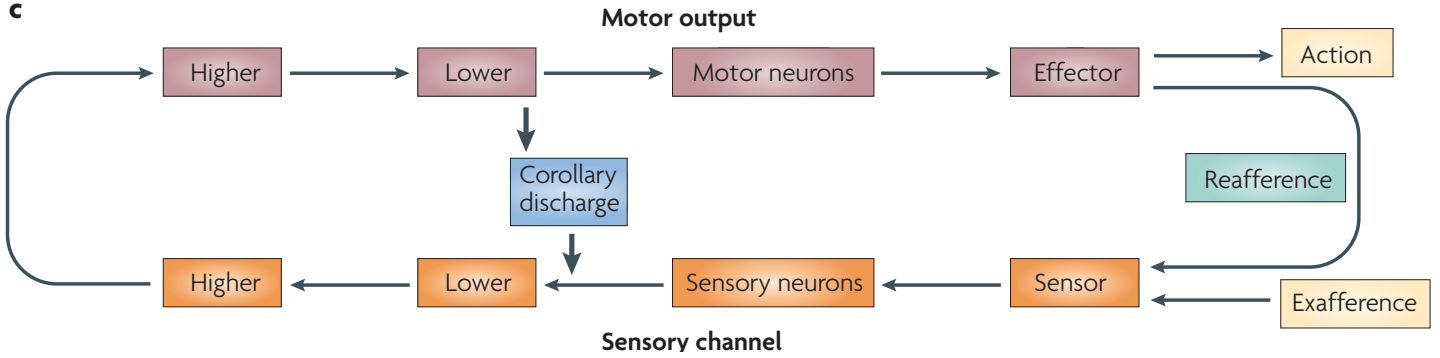

Figure 3 | Corollary discharge for reflex inhibition. a|The nematode Caenorhabditis elegans and a highly simplified schematic of the neural circuitry of its locomotor system. Activation of sensory neurons in one portion of C. elegans' body (either the front or the rear) results in the excitation of motor neurons that drive a movement away from the stimulus (backwards or forwards, respectively). Inhibitory connections silence the antagonistic pathway whenever one of these movements is elicited. Interneurons in the two sensorimotor circuits carry out corollary discharge (CD)-like functions (shown in blue). $\mathbf{b} \mid$ A member of the sea slug family Pleurobranchaea and a schematic diagram of portions of this family's nervous system, showing how CD during feeding suppresses withdrawal behaviour. During feeding, gastric information reaches $\mathrm{CD}$ interneurons (CDIs; shown in blue) from the feeding central pattern generators (CPGs). The CDIs inhibit the withdrawal command neurons (WCNs) and prevent tactile stimulation of the oral veil from triggering the retreat response. c|A basic schematic of the animals' CD circuitry is depicted on a common diagram. WMN, withdrawal motor neuron. 


\section{Box 1 | Vestibular and proprioceptive signals}

Corollary discharge (CD) signals have a role in regulating the sensation of position, velocity and acceleration that is mediated in part by the vestibular system and proprioception. The vestibular system, which is composed of the semicircular canals and otolith organs of the inner ear, helps to detect head motion and plays a critical part in maintaining balance. It also drives the vestibulocollic reflex. In response to a sudden displacement of the head, this reflex quickly restabilizes the head through activation of the neck musculature. There are times, however, when a vestibulocollic response is maladaptive, such as when an animal makes a willful attempt to move its head. It has been demonstrated in monkeys that $C D$ of head-movement commands suppresses neurons of the vestibular nucleus during voluntary head movement, thus preventing the inappropriate reflex response ${ }^{80}$. These $C D$ s permit differential processing of active and passive head movements by the vestibular nuclei, another case of $C D$ acting in the service of reflex inhibition.

Proprioceptors are found throughout the bodies of invertebrate and vertebrate organisms and provide continual information about body position and orientation. These receptors operate in static or dynamic contexts, signalling when parts of the body are at rest or are moving from one configuration to the next. It is in the latter context that $C D$ has a role. Inputs from proprioceptors must be regulated during active movement ${ }^{81}$ for two main reasons. If the proprioceptors are too sensitive, they could be triggered by self-movements and initiate inappropriate reflexes. If they are not sensitive enough, they could fail to protect the limb (for example, if an unexpected obstacle is struck). One type of proprioceptor, the muscle spindle, is regulated directly through projections from gamma motor neurons that receive $\mathrm{CD}$ of the movement commands sent to muscle-innervating alpha motor neurons ${ }^{82}$. More generally, $C D$ signals influence proprioceptive processing at multiple points in the neuraxis, ranging from the spinal cord ${ }^{83}$ to the cortex ${ }^{84}$.
Tympanate membrane

A thin membrane that detects sound (also known as the ear drum).

Gain

An input-output ratio that defines a neuron's responsiveness to incoming signals.
Sound-producing and -receiving organs allow organisms to exploit the physical characteristics of the surrounding environment by inducing and detecting pressure fluctuations. Among the many purposes of sound for animals is communication between conspecifics $^{21,22}$. A serious problem that is faced by organisms that are equipped with organs of sound production, however, is the substantial input that their sonic emissions impart to their auditory sensors. Following a sonic event, the auditory circuits could be overwhelmed; this would render the animal temporarily deaf to independently incoming sounds. CD mechanisms devoted to sensory filtration have evolved concurrently with the motor and sensory systems that are involved in acoustic communication. The solution for this problem seems to be ubiquitous, as evidenced by the examples of the cricket and the marmoset. Despite these animals occupying very different niches, they both adopted the same solution: tight coordination between their auditory and vocal systems by phasic CD that minimizes auditory reafference.

Crickets (see FIG. 4b) chirp by rubbing their forewings together, a process that is known as stridulation ${ }^{23}$. They hear through a tympanate membrane on their forelegs that is only millimeters from the site of stridulation ${ }^{24}$. A cricket's auditory system must therefore deal with a steady barrage of acoustic signals generated by the chirping. To prevent desensitization and ensure maximal attunement to environmental events, the cricket selectively screens the signals that enter the system. A CDI that performs this sensory filtration operation has been identified $^{25}$ (FIG. 4b). Positioned at the anatomical interface of the auditory and motor systems (an arrangement that is optimal for lower-motor to sensory-neuron contact), the cricket CDI is well suited to perform selective filtration operations that are coupled to motor activity. Intracellular recordings revealed that the CDI fires nearly simultaneously with wing motor-neuron bursts and exerts a phase-locked inhibitory influence on the interneurons of the auditory system ${ }^{25}$. By rhythmically imposing and lifting a blockade in phase with the song cycle, the cricket can chirp and remain receptive to the auditory environment.

Marmosets encounter the same problem as crickets: in principle, the sounds that they make should affect their hearing ${ }^{26}$. A protective mechanism is observable in the marmoset primary auditory cortex, where many neurons are suppressed during self-vocalizations ${ }^{27,28}$. Suppression begins $\sim 200$ ms before vocalization and continues for its duration. Both the predictive nature of the effect and the dependence on self-vocalization implicate CD as the cause of the suppression. In primates there are reciprocal connections between the primary auditory cortex and motor regions of the cerebral cortex ${ }^{29-31}$, and activity in the frontal cortex can precede vocalization by up to $1 \mathrm{~s}$ (REF. 32). Because the suppression begins $\sim 200 \mathrm{~ms}$ before the vocalization, the effect is most likely mediated by direct, rather than indirect, inhibition from one of these areas. As such, this could be a case in which CD interconnects motor and sensory areas that occupy comparable tiers of a sensorimotor pathway.

As with the sense of hearing, the sense of vision could theoretically be severely compromised by an animal's behaviour. However, audition and vision differ with respect to the nature of the motor interference. In audition, problems arise from reafferential acoustic noise. In vision, problems arise not from autogenic energy (energy originating from the animal itself - for example, the sound that results from a vocalization) but from the actions of the animal. If the eyes or the head move, the retinas go along for the ride. The retinas contain sheets of photoreceptors that are passively responsive to patterns of impinging photons. The moving of the retinas - called a gaze shift - enables an animal to sample a new part of visual space but, as we shall see, every gaze shift has its costs.

A gaze shift can be achieved with a saccadic (quick and discrete) eye movement and/or with a saccadic head movement. Saccadic gaze shifts cause, at least in principle, two serious reafferential problems. The first is that the speed of the movement could transiently blur the image. The second is the general displacement of the visual scene from before to after the movement, which could cause the scene to seem like it is jumping from one place to another.

The transient blur that occurs during saccades is minimized by a process called saccadic suppression ${ }^{33}$. In this process, a CD of the gaze shift lowers the gain of target visual structures to reduce information transfer between visual areas ${ }^{33}$. Saccadic suppression mechanisms have been identified in a host of different species, such as pigeons, chickens, locusts, monkeys and cats $^{34-38}$. Although the suppressive systems of these species differ with respect to the anatomical structures 
concerned, each circuit involves a CD operating as a gate that regulates signal traffic from the periphery - by definition a lower-order CD function. The second problem caused by saccades, the sudden jump of the visual scene, seems to be solved by other mechanisms (discussed below).

In the examples described up to now, the CD arises from a range of motor levels but generally targets peripheral sensory levels. In the cockroach, the crayfish and the cricket, the CD circuits discussed connect the lower motor tier to sensory neurons at or near the periphery. Overall, it seems that lower-order CD has a common functional impact: transient, protective inhibition of sensory networks. Next we turn to higher-order CD, the other broad taxonomic category of function.

\section{Higher-order CD}

Higher-order CD circuits are those that enable predictive control for perceptual cohesion and action sequencing. CD for perceptual cohesion allows an organism to move and yet continue to experience the world as it is (stable and continuous) rather than as it is sensed at the receptor level (in a chaotic and piecemeal fashion). CD permits specific brain structures to carry out appropriate adjustments in anticipation of the sensory input, and enables them to construct a cohesive representation of the world. The adjustments involve changes to neuronal processing, such as sudden shifts in sensory sensitivity (an example is described below). In addition, animals can string together movements with great speed, such that future movements are planned before prior a Sensory filtration

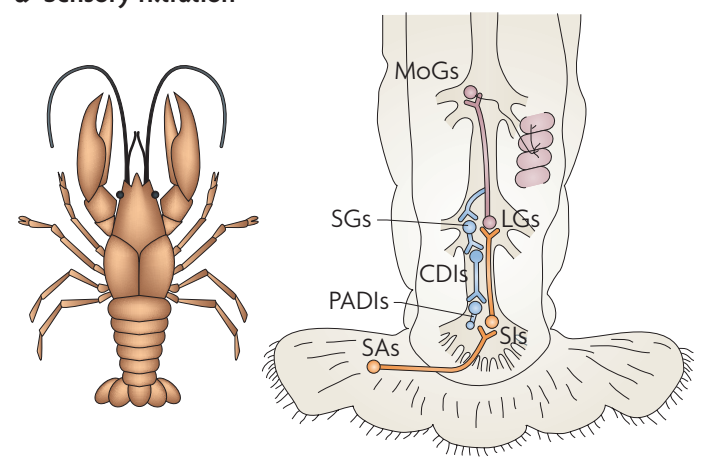

b

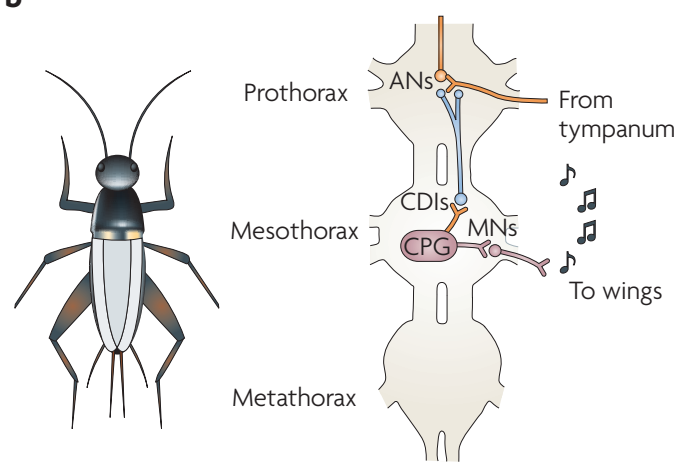

c

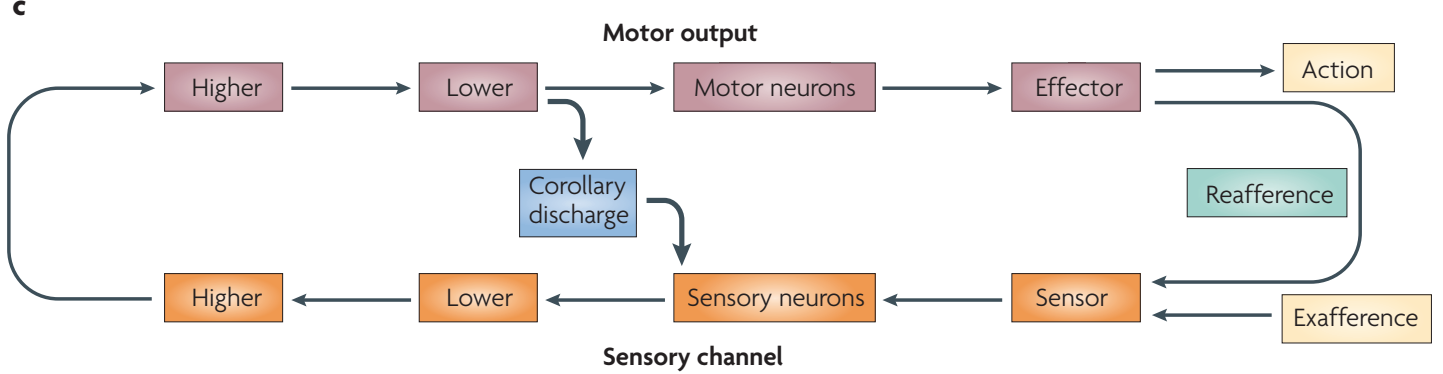

Figure 4 | Corollary discharge used for sensory filtration. a | The illustration on the left is of a crayfish species, Procambarus clarkii. The crayfish escapes from potential threats by producing a rapid tail-flip response. Mechanoreceptors lining its abdomen detect events in the water column and elicit the escape behaviour. Corollary discharge (CD) signals of movement commands protect the afferents to the mechanosensory escape system from maladaptive activation and desensitization. The schematic on the right depicts the circuitry that is involved in producing the crayfish's tail-flip response. Mechanical information enters the system through sensory afferents (SAs) and reaches the lateral giants (LGs) by way of sensory interneurons (SIs). The LGs communicate with segmental giants (SGs) and movement generators (MoGs) that activate flexor muscles in the abdomen. CD interneurons (CDIs) are activated by the SGs and convey signals to primary afferent depolarization interneurons (PADIs) that inhibit the SAs. This transient inhibition briefly silences the mechanosensory pathway and prevents tail-flip-induced reafference from generating further tail flips. Part c shows a circuit that represents an example of CD signals that originate from a lower motor cortical area and target sensory neurons at an early stage of the processing stream. $\mathbf{b}$ |The illustration on the left is of a cricket species, Gryllus bimaculatus. Crickets communicate with one another by chirping. Chirps are generated by rubbing their forewings together, a process that is known as stridulation. CDs of the wingbeats phasically inhibit components of the auditory system and prevent saturation and desensitization. The schematic of the cricket thorax on the right depicts how CD coordinates the 'song' and auditory systems. The activity of the central pattern generator (CPG) drives the wing motor neurons (MNs) that produce song (musical notes). Signals are routed concurrently from the CPGs to the CDIs. Collaterals of the CDIs synapse on to the axon terminals of auditory primary afferents and on to the cell bodies of auditory interneurons (ANs) in the prothorax. CPG-induced CDI activity rhythmically imposes and lifts an inhibitory gate on the auditory systems and restricts auditory traffic from the tympanum. Part $\mathbf{c}$ shows how, for the system in part $\mathbf{b}$, the point of contact between the motor and sensory systems is at the lower levels of the idealized sensorimotor circuit. 


\section{Box 2 | Shifting receptive fields and corollary discharge}

Many neurons of the primate visual system alter their visual sensitivity at the time of a saccade (see figure, part a). Visual responsiveness shifts before the saccade from the neurons' current visual receptive fields (RFs) to the location (the future field; FF) where the RFs will rest after the saccade. Because this process enables the FF (in the figure, the pepper) to be sampled both before and after the saccade, it is thought to contribute to a percept of visual stability despite the jerkiness of saccadic eye movements. One brain area that contains neurons with this property is the frontal eye field (FEF), a cortical structure that is involved in vision and eyemovement control ${ }^{42}$. FEF neurons that shift their RFs emit their visual-evoked burst of spikes in the middle of the saccade, on average. Perceptually this is advantageous for two reasons. First, it ensures that there is no premature perception of the world shifting before the saccade. Second, it ensures that following the saccade the system does not have to wait for visual reafference to arrive, which can take tens of milliseconds. A CD pathway that ascends from the superior colliculus (SC) to the FEF through the medial dorsal nucleus of the thalamus (MD) provides important saccadic information that triggers the shift (see figure, part b). Inactivation of this pathway at its point of relay in the thalamus reduces the ability of these FEF neurons to shift their RFs (see figure, parts $b$ and $\mathbf{c}$ ). In part $\mathbf{C}$ of the figure, therefore, instead of sensitivity shifting from the apple to the pepper during the saccade, sensitivity largely remains at the apple. It is only after the saccade that the neuron is fully responsive to the pepper, just like any other neuron with a 'classical' receptive field. It has been proposed that this would have a considerable impact on perception during saccadic eye movements ${ }^{40}$. During inactivation, the visual scene would seem to jump with each saccade, a consequence of $C D$ impairment and shifting RF reduction. With the pathway disrupted, monkeys trained to detect visual motion during saccades should report that stationary visual stimuli move with each saccade. This would be the first direct test of the $\mathrm{CD} /$ shifting-RF visual-stability hypothesis. In parts $\mathbf{a}$ and $\mathbf{c}$ of the figure, the red dot denotes the fixation point.

\section{a Normal}

Fixation
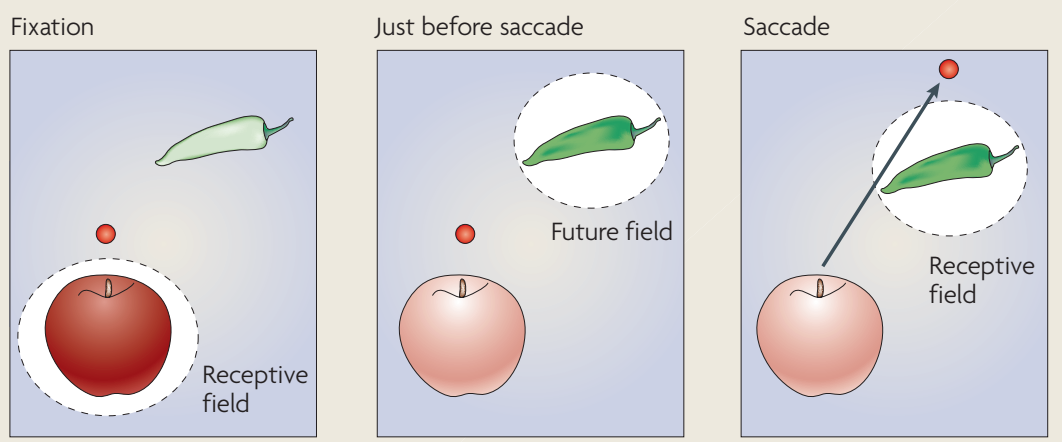

b Macaque brain

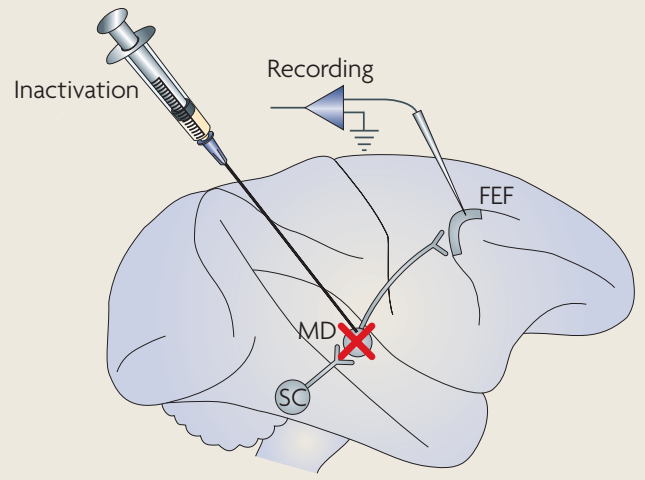

c During inactivation

Fixation

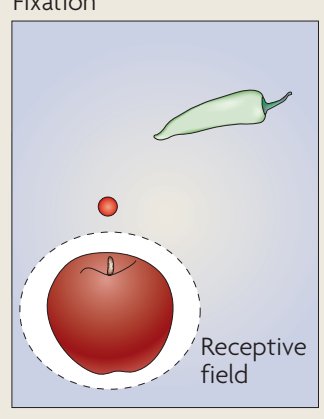

Just before saccade

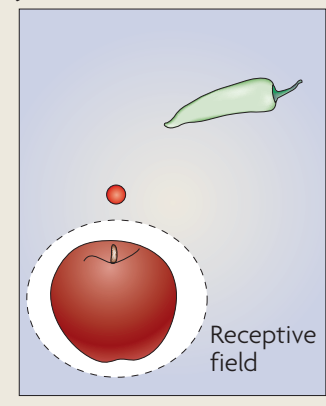

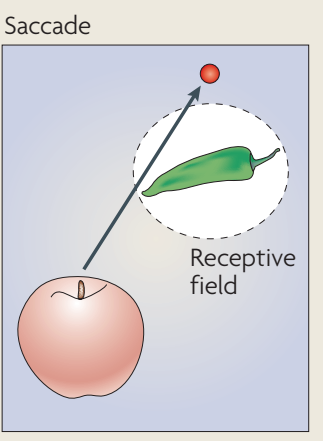

movements finish. Higher-order CD is crucial in this process of action sequencing because it provides internal feedback that escapes the afferent lags and bandwidth constraints of sensory or external feedback loops. Next we discuss how these principles are realized in various animals, first examining higher-order CDs devoted to sensory analysis and stability.

Sensory analysis and stability. As discussed above, in visuomotor behaviour, mechanisms of saccadic suppression that are mediated by lower-order CDs reduce the blur across the retinas. But even if blur were eliminated, a substantial problem would remain: the image on the retina is fully displaced by each saccade. Were we to perceive the world exactly as we sense it, the visual scene would seem to leap from place to place dozens of times per minute. It is crucial that the brain be able to distinguish what aspects of the 'jumpy' visual inflow are artefactual (due to saccades) as opposed to real (due to changes in the world). CD has long been thought to have a role in this process by providing advance warning of an imminent movement ${ }^{39}$. With this warning, the brain can enact a compensatory procedure to cancel the percept of visual-scene displacement that, in principle, should be perceived by an animal each time it moves its eyes. This is most problematic for species that frequently make saccades (the primate is a classic example).

In addition to $\mathrm{CD}$, there is another candidate mechanism for perceptual stability called spatial updating. Spatial updating involves pre-saccadic changes to a visual receptive field (RF). Although a typical RF is firmly retinotopic and samples a new part of the visual field (the 'new RF') only after the eye moves, a shifting $\mathrm{RF}$ is dynamic and starts sampling the new RF location even before a saccade (BOX 2). Because a saccade can have any direction and any amplitude, a neuron with a shift- 
ing $\mathrm{RF}$ requires the appropriate $\mathrm{CD}$ to calculate the new RF. At least some of this CD arises from a pathway that ascends from the midbrain ${ }^{40,41}$ to the frontal eye field, a cortical structure that is involved in visual processing and eye-movement control ${ }^{42}$. Interestingly, this same CD pathway assists in motor planning (discussed in the sensorimotor learning and planning section).

Similar perceptual issues hold for animals for which vision is a low priority. Rats explore the world largely by whisking tactile objects of interest ${ }^{43}$. How does the whisking rat construct a stable representation of the world from the variable and volatile inputs that flow in from the vibrissae? One clue to this puzzle was the identification of neural activity in the rat barrel cortex that was modulated by the act of whisking and was correlated with whisking amplitude ${ }^{44}$. The activity persisted during inactivation of the facial motor nerve to rule out proprioception. Because the signal was of central origin, a CD seems to have been at work. Such a signal could provide motor context to the barrel cortex and assist in the interpretation of vibrissal inputs, a step towards constructing a stable representation. Motor pathways are known to target the barrel cortex from the motor cortex, the superior colliculus and other brainstem areas ${ }^{45,46}$. Overall, the vibrissal system comprises a web of sensorimotor loops that span much of the neuraxis. Any of these loops could convey CD signals to the barrel cortex and thus mediate the effects observed in the above study ${ }^{44}$ and the assembly of coherent information from whisking events.

It is particularly intriguing to consider active sensing systems (those in which energy generated by an animal is used to probe the environment). Animals that are equipped with these exotic signalling modes exercise considerable control over the properties of the emitted signal (most of which are species- and modality-specific) and rely on complex predictive mechanisms for carrying out detailed analysis of the echo (the returned carrier signal $)^{47}$. Two common modes of active sensing are used by fish and bats, and CD is essential in both.

We first consider the mormyrid, a weakly electric fish (see FIG. 5Aa). The mormyrid uses self-generated electrical currents for communication and object identification. These currents flow from the mormyrid's electric organ into the surrounding water column and are analysed on their return by arrays of electroreceptors. A number of neural processes have evolved that allow the mormyrid to both extract the maximum information content from the return signal and avoid interference arising from reafference ${ }^{48}$.

Mormyrids sense the electromagnetic environment using electroreceptors (FIG. 5Aa). Primary afferents from these electroreceptors converge on layers of a medullary structure known as the electrosensory lateral line lobe (ELL) ${ }^{49}$. Separate mechanisms involving $\mathrm{CD}$ accommodate and protect the highly variegated electroreceptor systems ${ }^{50,51}$. For the ampullary system, an adaptable CD mechanism was found to be at work ${ }^{52}$. Each time a motor command exits the electric-organ command centre, a CD of the motor command also exits, targeting the ELL. One study dissociated reafferent responses of the ELL from effects of the electric-organ CD by pharmacologically decoupling the electricorgan motor command centre from the electric organ ${ }^{52}$. This preparation effectively silenced the electric organ: no electrical currents were produced. Nevertheless, the motor command and CD were still produced by the motor command centre. ELL neurons responded to the CD in a manner that was dependent on recent experience. If no external signals (that is, electrical current reafference) preceded the motor command, no response was elicited by the CD; however, if external electrical stimuli were paired with motor commands, responses that were opposite in sign and equal in duration to the stimulus interval gradually emerged. If external stimuli were removed, these responses were extinguished. The $\mathrm{CD}$ of the ampullary system is effectively a plastic photo negative of the expected reafference, and it varies as a function of recent sensory experience. A modifiable $\mathrm{CD}$ is evolutionarily advantageous because mormyrids navigate diverse aquatic environments that differ subtly in conductivity and resistivity. Interestingly, the mormyrid has two additional electroreceptor subtypes (mormyromasts and knollenorgans) that are susceptible to electric-organ discharge (EOD) feedback and, accordingly, compensatory CD systems are in place to accommodate them ${ }^{50,51}$. These systems differ from the ampullary system in that they require CDs that amplify (mormyromast) or inhibit (knollenorgan) the respective electroreceptor during EOD. As the mormyromast is specialized for electrolocation and the knollenorgan is specialized for conspecific communication, CDs for amplification and suppression, respectively, are appropriate. As the command centre and the ELL are located in the hindbrain, the CD is shown in FIG. $5 \mathrm{Ab}$ as linking lower motor and lower sensory levels.

Outside of aquatic environments, electrical probes have little utility. However, air is an excellent carrier of acoustic probes, a fact that is exploited by bats (see FIC. 5B). Bats explore the environment by emitting highintensity, high-frequency beams of sound and then comparing spatiotemporal aspects of the returning echo with the emitted sound ${ }^{53,54}$. Like the mormyrid, this active process of emission and comparison enables the bat to assemble an image of the external world ${ }^{55-57}$ and thus successfully navigate and predate.

Neurons that are sensitive to specific emission-echo intervals have been identified in the inferior colliculus (FIG. 5Ba) and, together with CD, have a key role in the neural circuitry for auditory-scene analysis ${ }^{54,58}$. The echo-sensitive neurons are thought to be primed by CDs of vocalizations that open time windows of echo analysis. If the echo returns within a specific time frame, then neurons that have been primed for analysis within that particular window will respond optimally and convey information to perceptual structures for further analysis. Such signals convey information that is related to the distance of approaching obstacles and the dimensions of environmental objects. These signals could arise from cortical or subcortical areas, so in FIG. 5Bb we represent the putative $\mathrm{CD}$ signals as emerging from both higher and lower motor levels. 
$\mathrm{Aa}$

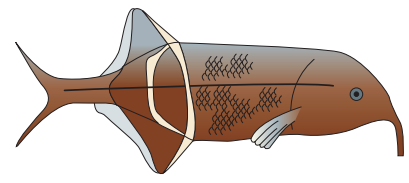

Mormyrid brain

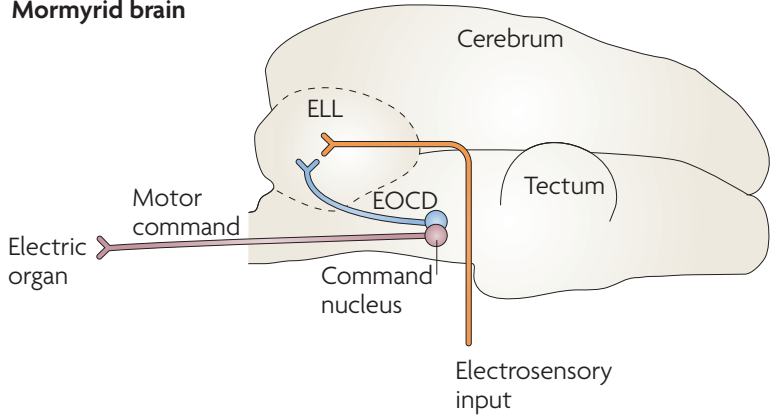

Ab

Motor output
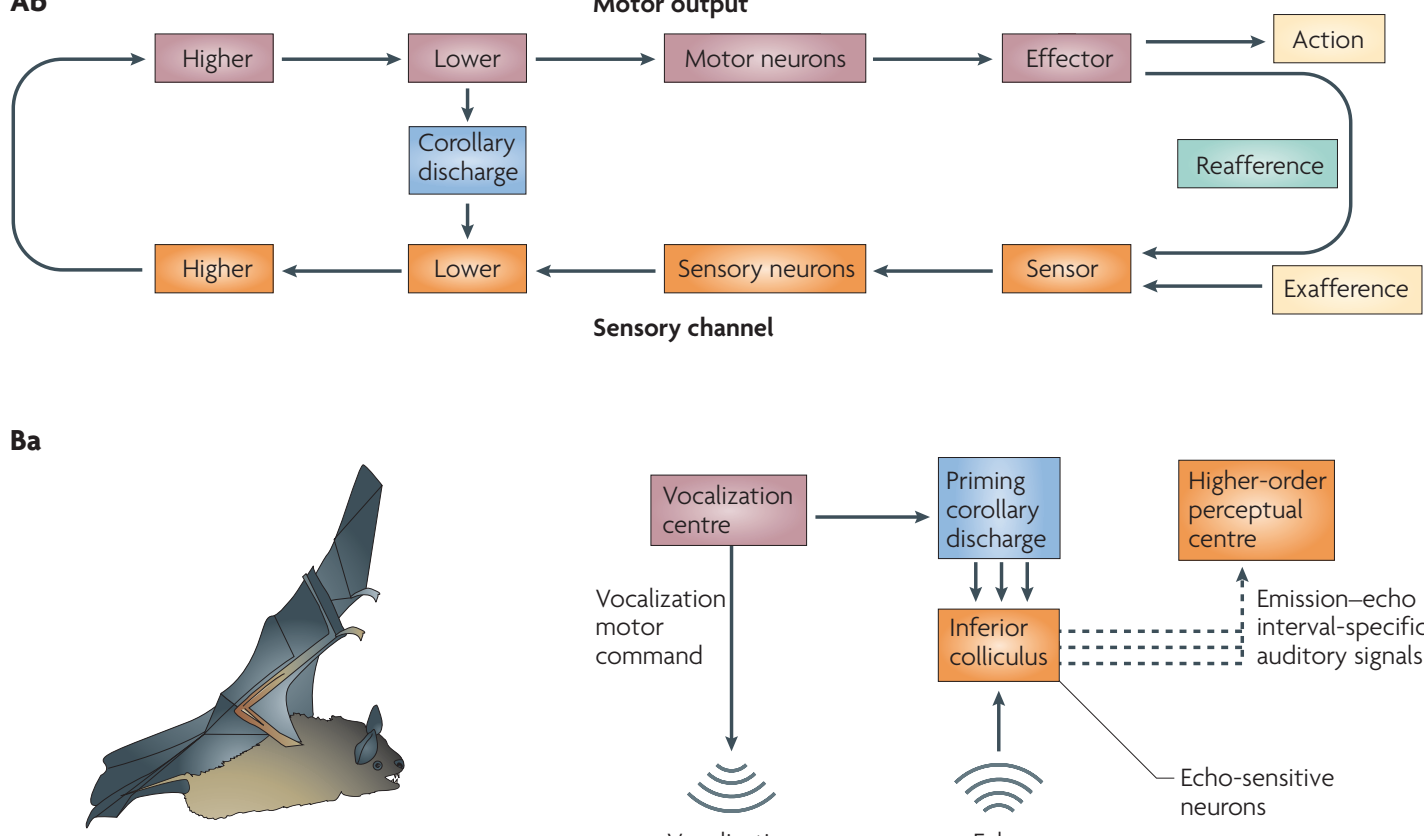

Sensory channel

\section{ns}

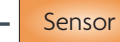

Exafference

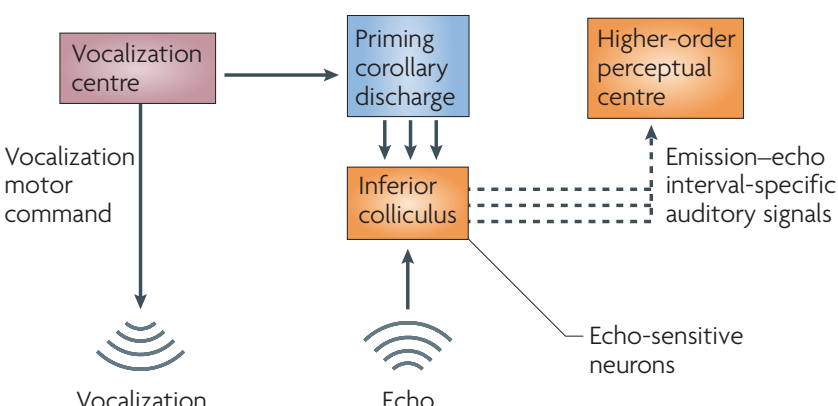

Bb

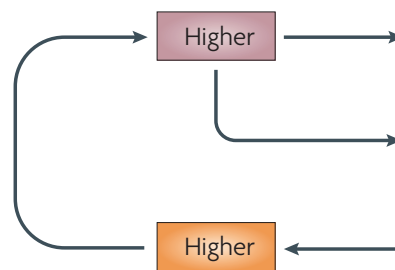

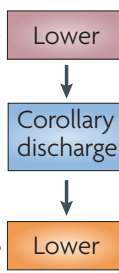

Motor output

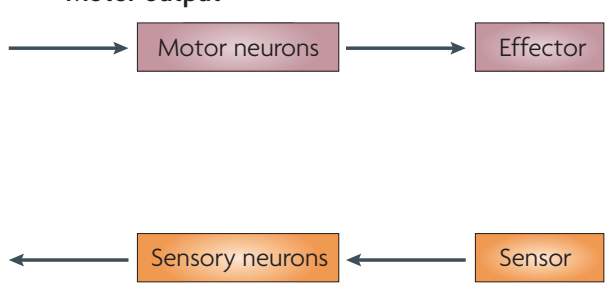

Sensory channel
Echo

\section{neurons}

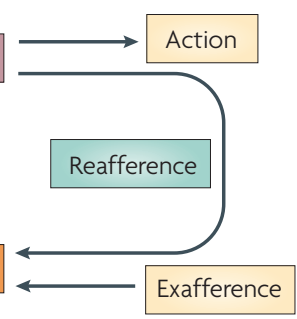

Figure 5 | Corollary discharge used for sensory analysis and stability. Aa | The illustration on the left is of the mormyrid species Gnathonemus petersii. The mormyrid generates electrical signals to probe the aquatic environment. Multiple types of corollary discharge (CD) of electrolocation commands allow the fish to gate, amplify or predict the return signal. The schematic of the mormyrid brain on the right illustrates pathways of the electrosensory system. Electric organ CD (EOCD; shown in blue) from the electric-organ motor command centre (which generates the motor command, shown in purple) reaches cellular networks of the electrosensory lateral line lobe (ELL), following which a host of interactions with electrosensory input (shown in orange) occur. $\mathbf{A b} \mid$ The $C D$ circuit that connects the command centre and the ELL is represented as a link between lower motor and lower sensory levels. Ba | The illustration on the left is of the bat species Rhinolophus rouxi. During high-speed flight, this bat uses sound to hunt. It compares a CD of the sonic probe to the measured echo to interpret the acoustic input. The schematic on the right shows how CD is used in this system. CD (shown in blue) represents the efferent motor command and innervates the inferior colliculus, where it is compared with the echo input. Differences between the $\mathrm{CD}$ and the input (shown as dashed lines) are analysed by higher-order centres to estimate the size, location and speed of the object that caused the echo. Bb | The CD signals could arise from any number of subcortical and cortical structures, corresponding to multiple pathways emerging from both higher and lower motor levels. 
In summary, these examples illustrate the diversity of sensory systems that are assisted by higher-order CD. In all of these different species, CDs aid the construction of an internal representation of the environment. We now consider motor planning and learning, the other category of higher-order $\mathrm{CD}$, in the saccading primate and vocalizing bird.

Sensorimotor learning and planning. Among the most stereotyped movements generated by primates are saccadic eye movements. As a primate looks around a scene, it must continuously update its internal record of the current saccade to facilitate planning the next. CD has repeatedly been implicated in this process ${ }^{59-64}$.

Electrophysiological recordings from a transiently inactivated circuit in the brain have shed some light on how $\mathrm{CD}$ is used for saccadic sequencing in rhesus monkeys ${ }^{62,63,65}$ (FIG. 6Aa). Neurons that connect the midbrain to the prefrontal cortex transmit bursts of action potentials that encode saccadic parameters. When this pathway was inactivated by muscimol, a GABA $(\gamma$-aminobutyric acid receptor type $A)$ agonist, behavioural deficits in the simplest saccadic sequencing task were observed. In this two-step task, the monkey was required to saccade to the locations of two rapidly flashed sequential targets. This particular task was chosen because it required the monkey to retrace the flash sequence without the aid of sensory feedback. The monkey had only the internal feedback provided by CD to inform it of its current eye position. If CD of the first eye movements were impaired by the inactivation, then the monkey would make incorrect second saccades owing to the absence of the feedback signal. The monkey would be oblivious to the execution of the first saccade and would perform the second saccade as if it had never made the first. The results conformed to this prediction, revealing a loss of CD. This is an example in which CD emerges from a lower-level motor structure and ascends the neuraxis to impinge on a higher-level executive centre (FIG. 6Ab). As discussed above and in BOX 2, this pathway also has a sensory analysis and stability function.

Vocalizations in birds are another prime example of action sequencing. Singing birds are thought to use updated, internal records of current phonations to generate subsequent phonations. Bilateral feedback pathways have been identified in the singing finch that might convey such vital information ${ }^{66}$. These pathways ascend from midbrain and medullary vocal-control nuclei up to the forebrain song system. Although their functional role in $\mathrm{CD}$ has not been tested directly using causal methods, they carry signals that are consistent with CDs of vocalization motor commands.

$\mathrm{CD}$ is especially important while vocalization is being learned. Male juvenile songbirds (see FIG. 6Ba) exhibit a stereotyped song that emerges gradually following several developmental stages ${ }^{67}$. The first stage is a sensory phase in which the juvenile bird listens to the songs of other adult birds known as tutors. The second stage consists of sensorimotor training in which the bird begins to sing spontaneously with syllables of varying intensity and motifs of high variability. The final phase is known as crystallization, and this is the phase in which mature song emerges. CD seems to have a key role during the second phase of vocal learning ${ }^{68,69}$. The bird is thought to fine-tune its song during this phase by comparing auditory feedback of its own vocalizations with copies of tutors' songs that are stored in memory. The errors between the memory and the actual feedback are corrected through an iterative cycle of vocal production, followed by adjustment of the motor programme. The bird has to keep track of its own motor commands in order to properly adjust and calibrate its subsequent commands. This is where CD plays a part. Intricate feedback loops that course throughout the avian song system are thought to convey the auditory, memory and CD signals ${ }^{70,71}$ (FIG. 6Ba). The feedback loops execute a delay function by increasing the distance over which $\mathrm{CD}$ that is issued from vocal centres travels before it collides with auditory feedback ${ }^{72,73}$. Evidence for this $\mathrm{CD}$ function was recently found in neurons in the high vocal centre (HVC) that fired with the same latency irrespective of whether the bird was actively singing or passively listening ${ }^{74}$. As the HVC is a premotor structure, the neurons would be expected to fire earlier for singing than for listening. The comparable latencies that were found for singing and passive listening imply that there is a delay function performed by a CD. Because a major site of comparison is the HVC, which is a higher brain structure of the auditory forebrain, in FIG. 6Bb we represent the $\mathrm{CD}$ as a signal between higher levels.

In these examples, $\mathrm{CD}$ is used for the higher-level functions of planning and learning actions. Accordingly, on exiting the motor domain, the CD signal impinges on higher-level structures that are highly sensory and/or executive in nature. As a result, appropriate behaviours can be prepared for the future (planning) and modified based on the lessons of the past (learning).

\section{Conclusions and future directions}

We have reviewed the operational impact of movement on sensation, and the ways in which nervous systems use CD signals to deal with movement-induced sensory problems. CD systems have evolved in tandem with the sensory systems of organisms that operate in diverse environments. The CD and the sensory systems are both shaped by the problems that are encountered by each organism, resulting in many different uses for CD signals. We have summarized these manifest differences with a functional taxonomy. After decades of experimentation, we now know a considerable amount about these $\mathrm{CD}$ circuits in both invertebrate and vertebrate organisms. In general, CD seems to be necessary for the proper function of nearly all sensory systems, and it exists in several guises at multiple levels in diverse species (perhaps all species). Some commonalities and subtle differences are apparent from the reviewed examples.

At one level (the lower-order level) CD is a discriminatory mechanism that prevents maladaptive responses and sensory saturation by restricting or filtering information. Functions such as reflex inhibition and sensory filtration are tightly controlled by this mode of signalling, 
$\mathrm{Aa}$

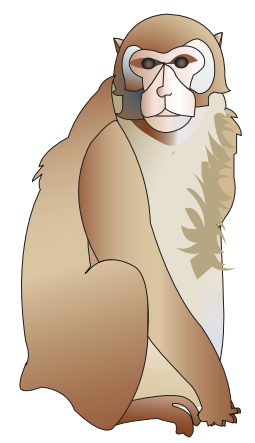

Macaque brain

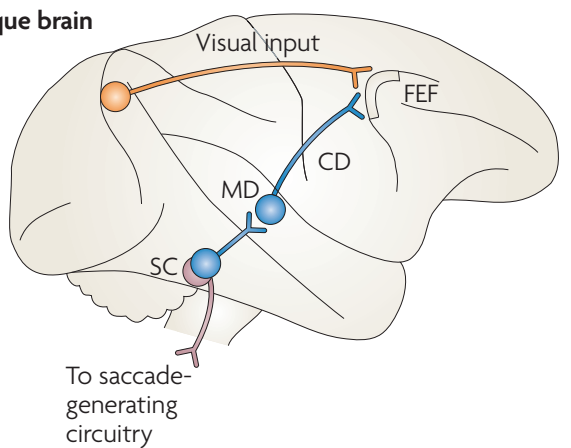

Ab

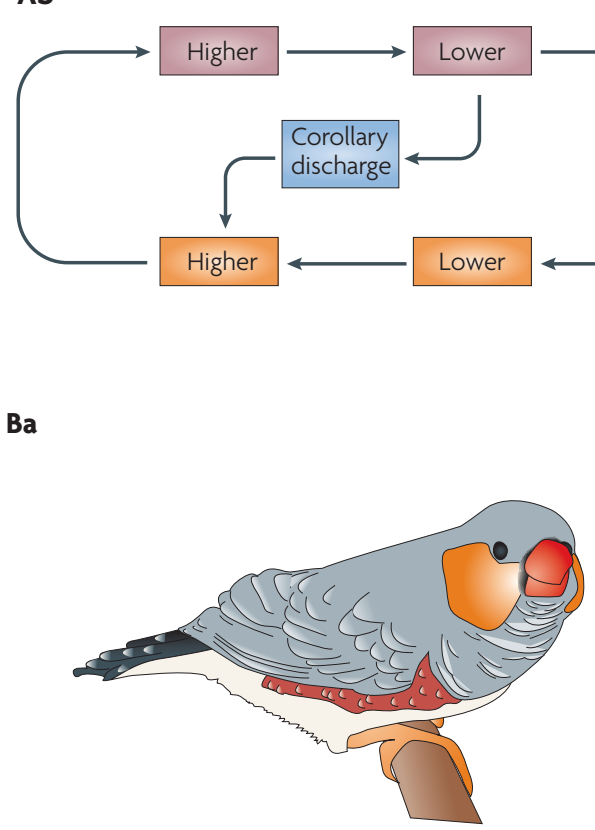

Motor output

Motor neurons
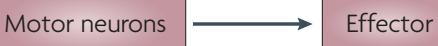

Effector

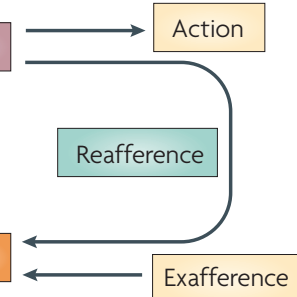

Sensory channel

Bb
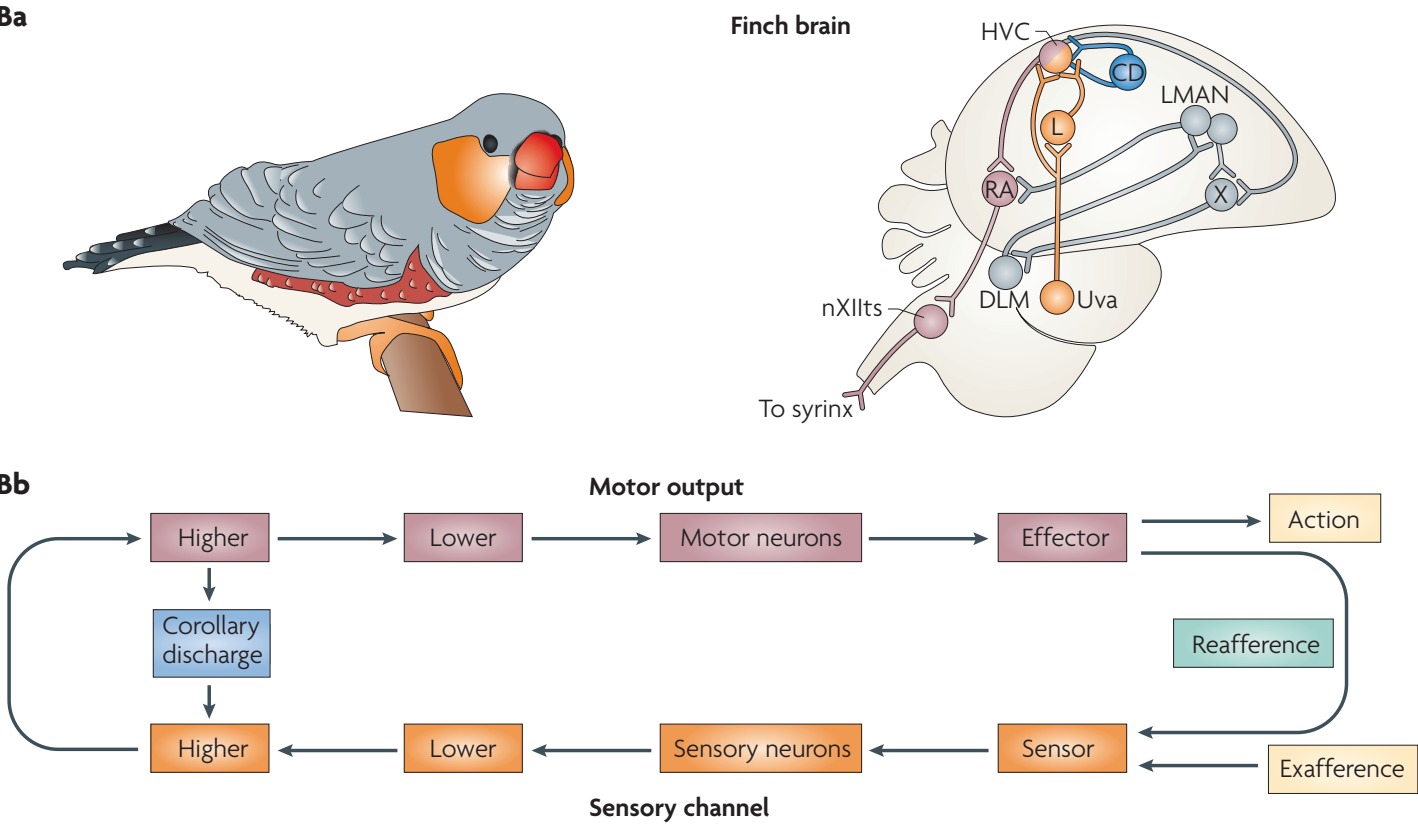

Figure 6 | Corollary discharge used for sensorimotor planning and learning. Aa|The illustration on the left is of the macaque monkey species Macaca mulatta. The macaque monkey visually explores its arboreal environment with rapid sequences of saccades. Corollary discharges (CDs) permit it to plan such sequences in rapid succession and enable it to predict the visual outcome for purposes of perceptual stability. The schematic of the macaque brain on the right illustrates the course of the $\mathrm{CD}$ (shown in blue): it ascends from the superior colliculus (SC) to the frontal eye field (FEF) by way of the medial dorsal nucleus of the thalamus (MD). Ab | This pathway is an example in which CD emerges from a lower-level motor area and targets a higher-level sensory area. Ba|The illustration on the left is of the songbird Poephila guttata (a finch species). The developing male finch progresses through a series of song-learning stages that conclude with the appearance of a mature, fully formed song. The schematic of the finch brain on the right depicts the circuitry and nuclei of the avian song-learning system. Intricate feedback loops that reside in the finch forebrain are involved in the songlearning process. CD has been proposed to course through some of these pathways and enable sensorimotor comparisons to occur within appropriate temporal windows. $\mathbf{B b} \mid$ The major site of comparison is proposed to reside in the forebrain; this CD pathway would correspond to contact between higher motor and sensory levels. DLM, medial nucleus of the dorsolateral thalamus; HVC, high vocal centre; L, field L; LMAN, lateral magnocellular nucleus of the anterior neostriatum; nXIlts, tracheo-syringeal portion of the hypoglossal nerve nucleus; RA, robust nucleus of the archistriatum; Uva, uvaeform nucleus of the thalamus; $\mathrm{X}$, area X. 
which is a type of access control. Computationally, the information content of these CD types is probably minimal, as they generally implement a gain mechanism that modulates a reflex or gates the inflow of sensory information at the periphery. As such, these signals seem to be one-dimensional in nature: time is the most crucial variable. This point is illustrated best in the nematode and gastropod examples of reflex inhibition. Here, CD intervenes at the precise time of the motor act to prevent an antagonistic reflex response. Timing is important too in the sensory-filtration examples. In the cricket, for instance, $\mathrm{CD}$ is synchronized rhythmically with singing to protect the auditory system. In short, each subtype of lower-order signal provides information about when the sensory input that is elicited from the motor act should occur. In other words, it is not so much what the signal says that matters, but when it is said.

We explained that lower-order CD circuits are illustrated best in invertebrates. The main characteristic of these circuits is the presence of a single interneuron, the CDI, which exerts inhibitory actions on various cellular components and controls interactions between motor and sensory circuitries through its suppressive influences. CDIs have been identified in Pleurobranchaea, crayfish and crickets. Suppressive influences by an inhibitory $\mathrm{CD}$ are also found in vertebrates, and it is tempting to speculate that in some species these operations could also be carried out by a type of CDI.

$\mathrm{CD}$ is also present in the animal kingdom as a higherorder mechanism that mediates sophisticated predictive computations. This type of CD underwrites complex behavioural phenotypes and cognitive operations, such as motor sequencing, sensorimotor learning and perceptual stabilization. Higher-order CD operates in the context of internal feedback circuitry, which involves multiple components and spans various levels of the neuraxis. Unlike CD that is involved with the central control of sensation, which is almost solely time-modulated, $\mathrm{CD}$ that is involved with the central control of action and perception carries information that represents a number of variables. In the monkey, for example, CD in the visuosaccadic system encodes spatial as well as temporal information about imminent saccades. The songbird's CD carries rich information too, particularly about song structure. In general, higher-order CDs are multidimensional and encode more parameters than just time. As we have described, the exact content and functional role of the signal (whether the $\mathrm{CD}$ is the prediction or whether it enables the recipient substrate to generate the prediction) varies from species to species and from system to system. But, in the end, what is said is just as important as when it is said. This architecture seems to be exclusive to higher vertebrates and might represent a later phylogenetic advancement that is particular to the demands of vertebrate life.

In all cases the corollary systems of remarkably diverse species could be easily accommodated into a common template that illustrates their underlying similarities. For the species that use reflex inhibition and sensory filtration, which are both cases of lower-order CD, the motor-to-sensory crossing points were schematically similar (that is, they were all lower-motor-area-tosensory-area neurons). Although the CD signals of the mormyrid and the bat, two sensory analysis/stability cases, emerged from differing motor tiers, their sensory-termination level was comparable (lower sensory areas). Similarly, the two illustrative sensorimotor species (a bird and a monkey) had different sites of motor emergence but common points of sensory termination (both terminated at higher sensory areas). An implicit assumption in this comparison is that the sensory target occupies increasingly higher tiers as one ascends from lower-order CD through the stages of higher-order CD (compare the nematode with the songbird). This illustrates the important point that although Sperry's original conception of CD matches the general flow of information from motor systems to sensory systems throughout the animal kingdom, it seems to be inappropriately simplistic to use a single term to describe the signal. There is no single type of CD - rather, there are numerous subtypes that correspond both to anatomical levels of the source and the target and, as we have emphasized, to functional utilities.

As a next step, one could break down the taxonomic subtypes even further. One way to do this would be to consider underlying mechanisms. Not all instantiations of $\mathrm{CD}$ are equal in their actions: $\mathrm{CD}$ signals can excite, inhibit or modulate their targets. Although finer-grained elaborations of the taxonomy would be beneficial, the point of the present Review was to synthesize all of the available data into what we see as the most essential functional taxonomy.

We are still at an early age of $C D$ research. Future studies should search for signs of CD in sensory domains that have been neglected, such as olfaction and gustation. There is evidence that components of the olfactory system are modulated by 'motor signals' during the act of sniffing ${ }^{75}$. Are these motor signals CDs? When animals masticate, do CDs modulate components of the gustatory system and thereby enhance taste discrimination? We should also further test the idea that aspects of cognition, such as thinking and decision making, use a type of CD for cohesion of self-identity ${ }^{76,77}$.

At the systems level, work should be devoted to understanding inter-areal circuits that mediate $\mathrm{CD}$ in the behaving animal. To date, only a few large-scale circuitlevel investigations of $\mathrm{CD}$ that combined behaviour with physiology have been performed ${ }^{38,40}$. We know quite a bit about the anatomical connections that probably convey $\mathrm{CD}$ - for example, a motor area 'A' that projects to a sensory area 'B'. But, until we introduce electrodes and record from these circuits during self-movements of the subjects, we can only speculate about their role in CD function.

At the more reductionist level, researchers should attempt to isolate CD mechanisms at ever-finer resolution, towards the cellular and molecular levels. The recent sequencing of various animal genomes is particularly exciting, as CD circuits could be manipulated genetically at various stages of an animal's life cycle. This could shed light on how CD circuits develop, an area that at present is poorly understood. 
Another outstanding question concerns the range of functional utilities of a given CD signal. A single $\mathrm{CD}$ circuit can mediate many different functions. One example we reviewed was from the visuosaccadic system of the primate ${ }^{40,62}$. In this system, a CD circuit has a role in sensorimotor planning as well as in sensory stability (compare FIG. 6A with BOX 2). What is the connection between these two differing processes and the $\mathrm{CD}$ circuit? Is there more than one CD signal coursing through the circuit, or is there a single, multi-purpose CD signal? Does behavioural context play a part in determining what computation is implemented by the $\mathrm{CD}$, and its functional interpretation by the recipient sensory structure? Similar CD complexities are likely to be present in other sensorimotor systems. Does the whisking rat use the same CD signal for sequencing its whisker movements as for constructing a stable tactile image of the whisked object? Or does it use different CD signals for each functional application? These perplexing questions remain to be addressed by further circuit-level interventions that attempt to disentangle the multifarious uses nervous systems have for CD signals.

Finally, the ultimate goal is to discover how CD influences perception. Experiments thus far have shown that inactivation of CD pathways can alter behaviour, and covert perceptual changes might accompany these behavioural changes. Eye movements in a two-step task, for example, change if CD about previous movements are impaired. Comparable experiments could be performed in other animals, such as rats that have been trained to whisk an object in a particular sequence. Do such animals, with their CD circuits interrupted, experience a sensory world that fractionates from stable to jumpy? How could we determine this? Although it is challenging to assess what an animal is perceiving, in rhesus monkeys, for example, it seems to be possible $\mathrm{e}^{78,79}$. Now that the basic layout of many CD circuits has been established, it will be exciting to manipulate the circuits while probing animals with careful psychophysical tests to determine how perception is altered.

Whatever is uncovered by future studies of CD, it seems safe to say that we can currently recognize at least one fundamental principle. All animals, from the humble nematode to the cognitively advanced primate, require the type of signalling that is enabled by $\mathrm{CD}$. In addition to the usual flow of information from sensory systems to motor systems, there is extensive signalling in the opposite direction by motor systems reporting their activities to sensory structures. It is this coordination between the two systems that makes it possible to analyse the world while moving within it.
1. Poincaré, H. Science et Methode (Flammarion, Paris, 1897).

2. Holst, E. V. \& Mittelstaedt, H. The reafference principle. Naturwissenschaften 37, 464-467 (1950).

3. Sperry, R. Neural basis of the spontaneous optokinetic response produced by visual inversion. J. Comp. Physiol. Psychol. 43, 482-489 (1950).

References 2 and 3 are two groundbreaking papers that were published independently and nearly simultaneously. They were the first to propose in a rigorous manner, and with supporting experimental evidence, that motor-to-sensory feedback has a critical role in regulating animal behaviour.

4. Cullen, K. E. Sensory signals during active versus passive movement. Curr. Opin. Neurobiol. 14, 698-706 (2004)

5. Poulet, J. F. \& Hedwig, B. New insights into corollary discharges mediated by identified neural pathways. Trends Neurosci. 30, 14-21 (2007).

6. White, J. G., Southgate, E., Thomson, J. N. \& Brenner, S. The structure of the nervous system of the nematode Caenorhabditis elegans. Philos. Trans. R. Soc. Lond. B Biol. Sci. 314, 1-340 (1986).

7. Rankin, C. H. Interactions between two antagonistic reflexes in the nematode Caenorhabditis elegans. J. Comp. Physiol. A 169, 59-67 (1991).

8. Chalfie, M. et al. The neural circuit for touch sensitivity in Caenorhabditis elegans. J. Neurosci. 5, 956-964 (1985).

9. Sillar, K. T. \& Roberts, A. A neuronal mechanism for sensory gating during locomotion in a vertebrate. Nature 331, 262-265 (1988)

10. Davis, W. J., Siegler, M. V. S. \& Mpitsos, G. J. Distributed neuronal oscillators and efference copy in the feeding system of Pleurobranchaea. J. Neurophysiol. 36, 258-274 (1973).

This was one of the first electrophysiological studies to characterize $C D$ signals at the cellular level.

11. Eaton, R. Neural Mechanisms of Startle Behavior (Plenum, New York, 1984)

12. Edwards, D., Heitler, W. \& Krasne, F. Fifty years of a command neuron: the neurobiology of escape behavior in the crayfish. Trends Neurosci. 22, 153-161 (1999).

13. Hatsopoulos, N., Gabbiani, F. \& Laurent, C. Elementary computation of object approach by a widefield visual neuron. Science 270, 1000-1003 (1995).
14. Levi, R. \& Camhi, J. Wind direction coding in the cockroach escape response: winner does not take all. Neuroscience 20, 3814-3821 (2000).

15. Krasne, F. B. \& Bryan, J. S. Habituation: regulation through presynaptic inhibition. Science $\mathbf{1 8 2}$, 590-592 (1973).

16. Delcomyn, F. Corollary discharge to cockroach giant interneurons. Nature 269, 160-162 (1977).

17. Kroese, A. B. A. \& van Netten, S. M. in The Mechanosensory Lateral Line: Neurobiology and Evolution (eds Coombs, S., Gorner, P. \& Munz, H.) 265-284 (Springer, New York, 1989).

18. Coombs, S. \& Montgomery, J. C. in Comparative Hearing: Fish and Amphibians (eds Fay, F. R. \& Popper, A. N.) 319-362 (Springer, New York, 1999).

19. Harris, G. G. \& van Bergeijk, W. A. Evidence that the lateral-line organ responds to near-field displacements of sound sources in water. J. Acoust. Soc. Am. 34, 1831-1841 (1962).

20. Roberts, B. L. \& Russell, I. J. The activity of lateral line efferent neurones in stationary and swimming dogfish. J. Exp. Biol. 57, 435-448 (1972).

21. Michelsen, A. in The Evolutionary Biology of Hearing (eds Webster, D. B., Fay, F. R. \& Popper, A. N.) 61-77 (Springer, New York, 1992).

22. Popper, A. N., Platt, P. \& Edds, P. in The Evolutionary Biology of Hearing (eds Webster, D. B., Fay, F. R. \& Popper, A. N.) 49-57 (Springer, New York, 1992).

23. Hedwig, B. Pulses, patterns, and paths: neurobiology of acoustic behavior in crickets. J. Comp. Physiol. A 192, 677-689 (2006)

24. Hoy, R. R. \& Robert, D. Tympanal hearing in insects. Annu. Rev. Entomol. 41, 433-450 (1996).

25. Poulet, J. F. A. \& Hedwig, B. The cellular basis of a corollary discharge. Science 311, 518-522 (2006)

This report is one of a series of elegant studies carried out by the authors in which they homed in on, and both anatomically and physiologically identified, a CDI in the cricket auditory system.

26. Agamaite, J. \& Wang, X. Quantitative classification of the vocal repertoire of the common marmoset (Callithrix jacchus jacchus). Assoc. Res. Otolaryngol. Abstr. 20, 573 (1997).

27. Eliades, S. J. \& Wang, X. Sensory-motor interaction in the primate auditory cortex during self-initiated vocalizations. J. Neurophysiol. 89, 2194-2207 (2003).
28. Eliades, S. J. \& Wang, X. Dynamics of auditory-vocal interaction in monkey auditory cortex. Cereb. Cortex 15, 1510-1523 (2005)

29. Alexander, G., Newman, J. \& Symmes, D. Convergence of prefrontal and acoustic inputs upon neurons in the superior temporal gyrus of the awake squirrel monkey. Brain Res. 116, 334-338 (1976).

30. Hackett, T., Stepniewska, I. \& Kaas, J. Prefrontal connections of the parabelt auditory cortex in macaque monkeys. Brain Res. 817, 45-58 (1999).

31. Morel, A. \& Kaas, J. Subdivisons and connections of auditory cortex in owl monkeys. J. Comp. Neurol. 318, 27-63 (1992)

32. Gemba, H., Miki, N. \& Sasaki, K. Cortical field potentials preceding vocalization and influences of cerebellar hemispherectomy upon them in monkeys. Brain Res. 697, 143-151 (1995).

33. Ross, J., Morrone, M. C., Goldberg, M. E. \& Burr D. C. Changes in visual perception at the time of saccades. Trends Neurosci. 24, 113-121 (2001)

34. Marin, G., Letelier, J. C. \& Wallman, J. Saccade-related responses of centrifugal neurons projecting to the chicken retina. Exp. Brain Res. 82, 263-270 (1990)

35. Zaretsky, M. \& Rowell, C. H. F. Saccadic suppression by corollary discharge in the locust. Nature $\mathbf{2 8 0}$, 583-584 (1979).

36. Thiele, A Henning P. Kubischik, M. \& Hoffmann, K. P. Neural mechanisms of saccadic suppression. Science 295, 2460-2462 (2002).

37. Lee, D. \& Malpeli, J. G. Effects of saccades on the activity of neurons in the cat lateral geniculate nucleus. J. Neurophysiol. 79, 922-936 (1998).

38. Yang, Y., Cao, P., Yang, Y. \& Wang, S. R. Corollary discharge circuits for saccadic modulation of the pigeon visual system. Nature Neurosci. 11, 595-602 (2008).

39. von Helmholtz, H. Helmholtz's Treatise on Physiological Optics (Optical Society of America, New York, 1925).

40. Sommer, M. A. \& Wurtz, R. H. Influence of the thalamus on spatial visual processing in frontal cortex. Nature 444, 374-377 (2006).

41. Sommer, M. A. \& Wurtz, R. H. Brain circuits for the internal monitoring of movements. Annu. Rev. Neurosci. 31, 317-338 (2008).

42. Schall, J. D. On the role of frontal eye field in guiding attention and saccades. Vision Res. 44, 1453-1467 (2004). 
43. Kleinfeld D. Ahissar, E. \& Diamond, M. E. Active sensation: insights from the rodent vibrissa sensorimotor system. Curr. Opin. Neurobiol. 16 435-444 (2006)

44. Fee, M. S., Mitra, P. P. \& Kleinfeld, D. Central versus peripheral determinants of patterned spike activity in rat vibrissa cortex during whisking. J. Neurophysiol. 1997, 1144-1149 (1997).

45. Ahissar, E. \& Kleinfeld, D. Closed-loop neuronal computations: focus on vibrissa somatosensation in rat Cereb. Cortex 13, 53-62 (2003).

46. Kleinfeld, D., Berg, R. W. \& O'Conner, S. M Anatomical loops and their electrical dynamics in relation to whisking by rat. Somatosens. Mot. Res. 16, 69-88 (1999)

47. Nelson, M. E. \& Maclver, M. A. Sensory acquisition in active sensing systems. J. Comp. Physiol. A 192, 573-586 (2006)

48. Caputi, A. A. Contributions of electric fish to the understanding of sensory processing by reafferent systems. J. Physiol. (Paris) 98, 81-97 (2004).

49. Meek, J., Grant, K. \& Bell, C. Structural organization of the mormyrid electrosensory lateral line lobe. J. Exp. Biol. 202, 1291-1300 (1999).

50. Mohr, C., Roberts, P. D. \& Bell, C. C. The mormyromast region of the mormyrid electrosensory lobe. I. Responses to corollary discharge and electrosensory stimuli. J. Neurophysiol. 90 , 1193-1210 (2003)

51. Bell C C. \& Grant K Corollary discharge inhibition and preservation of temporal information in a sensory nucleus of mormyrid electric fish. J. Neurosci. $\mathbf{9}$, 1029-1044 (1989).

52. Bell, C. C. An efference copy which is modified by reafferent input. Science 214, 450-453 (1981). This was a pioneering study that unveiled a plastic $\mathrm{CD}$ in the mormyrid that was modifiable by recent sensory experience.

53. Moss, C. F. \& Sinha, S. R. Neurobiology of echolocation in bats. Curr. Opin. Neurobiol. 13 751-758 (2003)

54. Neuweiler, G. Evolutionary aspects of bat echolocation. J. Comp. Physiol. A 189, 245-256 (2003).

55 Simmons, J. A A view of the world through the bat's ear: the formation of acoustic images in echolocation. Cognition 33, 155-199 (1989).

56. Simmons, J. A. \& Kick, S. A. Physiological mechanisms for spatial filtering and image enhancement in the sonar of bats. Annu. Rev. Physiol. 46, 599-614 (1984).

57. Simmons, J. A. et al. in Hearing by Bats (eds Fay, F. R \& Popper, A. N.) 146-190 (Springer, New York, 1995).
58 Schuller, G. Vocalization influences auditory processing in collicular neurons of the CF-FM bat, Rhinolophus ferrumequinum. J. Comp. Physiol. A 132, 39-46 (1979).

59. Bellebaum, C., Daum, I., Koch, B., Schwarz, M. \& Hoffmann, K. P. The role of the human thalamus in processing corollary discharge. Brain 128 , 1139-1154 (2005)

60. Bellebaum, C., Hoffmann, K. P., Koch, B., Schwarz, M. \& Daum, I. Altered processing of corollary discharge in thalamic lesion patients. Eur. J. Neurosci. 24 2375-2388 (2006)

61. Guthrie, B. L., Porter, J. D. \& Sparks, D. L. Corollary discharge provides accurate eye position information to the oculomotor system. Science 221, 1193-1195 (1983)

62. Sommer, M. A. \& Wurtz, R. H. A pathway in primate brain for internal monitoring of movements. Science 296, 1480-1482 (2002).

This was the first study to identify a CD pathway in the primate brain.

63. Sommer, M. A. \& Wurtz, R. H. What the brain stem tells the frontal cortex. II. Role of the SC-MD-FEF pathway in corollary discharge. J. Neurophysiol. 91 , 1403-1423 (2004)

64. Tanaka, M. Inactivation of the central thalamus delays self-timed saccades. Nature Neurosci. 9, 20-22 (2006)

65. Lynch, J., Hoover, J. \& Strick, P. Input to the primate frontal eye field from the substantia nigra, superior colliculus, and dentate nucleus demonstrated by transneuronal transport. Exp. Brain Res. 100 181-186 (1994).

66. Striedter, G. F. \& Vu, E. T. Bilateral feedback projections to the forebrain in the premotor network for singing in zebra finches. J. Neurobiol. 34, 27-40 (1998).

67. Catchpole, D. K. \& Slater, P. J. B. Bird Song: Biologica Themes and Variations (Cambridge Univ. Press, Cambridge, 1995).

68. Brainard, M. \& Doupe, A. J. Auditory feedback in learning and maintenance of vocal behaviour. Nature Rev Neurosci. 1, 31-40 (2000)

69. Margoliash, D. Evaluating theories of bird song learning: implications for future directions. J. Comp. Physiol. A 188, 851-866 (2002).

70. Margoliash, D. Functional organization of forebrain pathways for song production and perception. J. Neurobiol. 33, 671-693 (1997).

71. Reiner, A., Yamamoto, K. \& Karten, H. Organization and evolution of the avian forebrain. Anat. Rec. A Discov. Mol. Cell. Evol. Biol. 287, 1080-1102 (2005).

72. Troyer, T. W. \& Doupe, A. J. An associational model of birdsong sensorimotor learning I. Efference copy and the learning of song syllables. J. Neurophysiol. 84 1204-1223 (2000).
73. Troyer, T. W. \& Doupe, A. J. An associational model of birdsong sensorimotor learning II. Temporal hierarchies and the learning of song sequence. J. Neurophysiol. 84, 1224-1239 (2000).

74. Prather, J. F., Peters, S., Nowicki, S. \& Mooney, R. Precise auditory-vocal mirroring in neurons for learned vocal communication. Nature 451, 305-310 (2008).

75. Mainland, J. \& Sobel, N. The sniff is part of the olfactory percept. Chem. Senses 31, 181-196 (2006).

76 Feinberg I. \& Guazzelli, M. Schizophrenia-a disorder of the corollary discharge systems that integrate the motor systems of thought with the sensory systems of consciousness. Br. J. Psychiatry 174, 196-204 (1999).

77. Ford, J. M. et al. Neurophysiological evidence of corollary discharge dysfunction in schizophrenia. Am. J. Psychiatry 158, 2069-2071 (2001).

78. Logothetis, N. K. Single units and conscious vision. Philos. Trans. R. Soc. Lond. B Biol. Sci. 353. 1801-1818 (1998).

79. Parker, A. J. \& Newsome, W. T. Sense and the single neuron: probing the physiology of perception. Annu. Rev. Neurosci. 21, 227-277 (1998).

80. Roy, J. E. \& Cullen, K. E. Dissociating self-generated from passively applied head motion: neural mechanisms in the vestibular nuclei. J. Neurosci. 24, 2102-2111 (2004)

81. Rossignol, S., Dubuc, R. \& Gossard, J. P. Dynamic sensorimotor interactions in locomotion. Physiol. Rev. 86, 89-154 (2006).

82. Matthews, P. B. C. Where does sherrington's "muscular sense" originate? Muscles, joints, corollary discharges? Annu. Rev. Neurosci. 5, 189-218 (1982).

83 Seki, K. Perlmutter S. I. \& Fetz, E. E. Sensory input to primate spinal cord is presynaptically inhibited during voluntary movement. Nature Neurosci. 6 . 1309-1316 (2003)

84. Voss, M., Ingram, J. N., Haggard, P. \& Wolpert, D. M Sensorimotor attenuation by central motor command signals in the absence of movement. Nature Neurosci. 9, 26-27 (2006)

Acknowledgements

We thank R. H. Wurtz for comments on an earlier version of the manuscript. Supported by the Alfred P. Sloan foundation and RO1-EY017592 to M.A.S.

FURTHER INFORMATION

Marc Sommer's homepage: http://cnup.neurobio.pitt.edu/ people/peopleDetail.aspx?uid=381

ALL LINKS ARE ACTIVE IN THE ONLINE PDF 